\title{
Sex differences in thoracic aortic disease: $A$ review of the literature and a call to action
}

Jennifer Chung, MD, MSC, ${ }^{\mathrm{a}}$ Thais Coutinho, MD, ${ }^{\mathrm{b}}$ Michael W. A. Chu, MD, MEd, ${ }^{\mathrm{c}}$ and Maral Ouzounian, $\mathrm{MD}, \mathrm{PhD}^{\mathrm{a}}$

There has been increasing recognition of important sex differences in the development, management, and outcomes of cardiovascular diseases. Identifying and understanding why sex differences exist is a crucial first step toward reducing disparities observed in clinical outcomes between women and men. To this end, leading medical journals and scientific bodies, including the National Institutes of Health, have called for a systematic and mandatory approach to reporting sex and gender in all publications, clinical trials, and grant applications.

The cardiovascular literature has repeatedly demonstrated delayed diagnosis, suboptimal management, and worse outcomes following medical and surgical treatment in women compared with men. These findings have been observed in patients across every form of cardiovascular disease, including coronary disease, valvular pathology, thoracic and abdominal aortic aneurysms, and cerebrovascular and peripheral vascular disease. Several hypotheses have been raised to explain these disturbing and consistent findings; however, the mechanisms behind these observations remain poorly understood.

This review summarizes the contemporary literature regarding sex differences in thoracic aortic aneurysm disease. We describe differences in the epidemiology, biology, and natural history of thoracic aortic aneurysm and dissection (TAAD), as well as surgical management and outcomes. We propose several explanations for these observed differences and suggest a call to action to better understand and close the gap in outcomes for women with TAAD.

\section{Sex Differences in the Epidemiology, Biology, and Natural History of TAAD}

Aortic aneurysm is an indolent, silent, yet virulent disease associated with high morbidity and mortality. Data from developed countries show that thoracic aortic

\footnotetext{
From the a Division of Cardiac Surgery, Department of Surgery, Peter Munk Cardiac Centre, University of Toronto, Toronto, Ontario; ${ }^{b}$ Division of Cardiology, Ottawa Heart Institute, University of Ottawa, Ottawa, Ontario; and ${ }^{\mathrm{c}}$ Division of Cardiac Surgery, Department of Surgery, Western University, London, Ontario, Canada.

Received for publication July 24, 2019; revisions received Sept 8, 2019; accepted for publication Sept 11, 2019; available ahead of print Jan 24, 2020.

Address for reprints: Maral Ouzounian, MD, PhD, Peter Munk Cardiac Centre, 200 Elizabeth St, 4N-464, Toronto, ON, Canada M5G 2C4 (E-mail: Maral. ouzounian@uhn.ca).

J Thorac Cardiovasc Surg 2020;160:656-60 0022-5223/ $\$ 36.00$

Copyright $₫ 2020$ Published by Elsevier Inc. on behalf of The American Association for Thoracic Surgery

https://doi.org/10.1016/j.jtcvs.2019.09.194
}

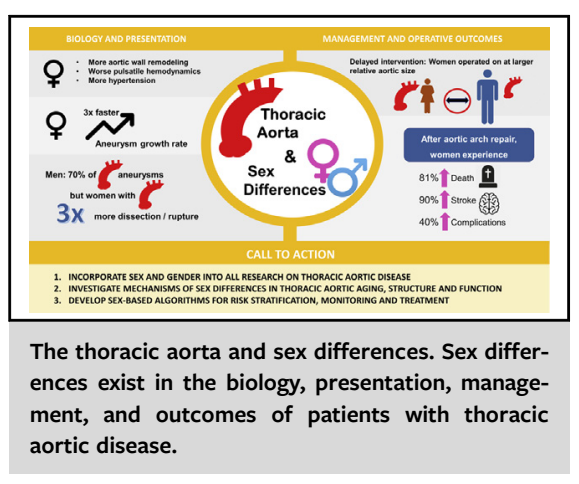

CENTRAL MESSAGE

Important sex-related differences exist in the epidemiology, biology, management, and outcomes of patients with thoracic aortic disease.

The Invited Expert Opinion provides a perspective on this topic based on the following paper: Circulation. 2019;139:1177-1184. https://doi.org/10. 1161/CIRCULATIONAHA.118.035805.

disease affects nearly $0.16 \%$ of the population, with a yearly incidence of aortic aneurysms of 10.4/100,000 and of acute aortic syndromes of $7 / 100,000 .{ }^{1}$ However, these numbers are undoubtedly underestimated, given that reports rely heavily on acute aortic syndromes and not the entire TAAD population. As many as $22 \%$ of people who experience an acute dissection die at home before receiving medical attention, and among those who reach the hospital alive, $34 \%$ die within the first 30 days. ${ }^{1}$

Up to $25 \%$ of patients with TAAD have evidence of a heritable aortopathy, most often due to a highly penetrant pathogenic variant that is inherited in an autosomal dominant manner. Although men and women are equally likely to inherit the predisposition, individuals with single gene disorders predisposing to TAAD are predominantly men. Even among patients with syndromic disorders, there exist differential aortic risk profiles, including age of onset, aortic presentation, and outcomes based on sex. In the largest cohort reporting the association of sex with phenotypic features of Marfan syndrome, males were more likely to have aortic root dilatation and aortic regurgitation and to have undergone surgery than females $(P<.001$ for all $)$, 
indicating more severe and earlier manifestations of aortic disease in men., ${ }^{2,3}$ In contrast, in a study of aortic growth rate and the risk of acute dissection in Marfan syndrome, individuals who were "fast growers" had a $25 \%$ dissection rate, with disproportionately more women affected. ${ }^{4}$ Men with TGFBR1 mutations have more aggressive aortic disease compared with women, with earlier age of first aortic event and more aortic dissections. ${ }^{5}$ In contrast, these sexbased differences were not observed for patients harboring pathogenic variants in TGFBR2 or SMAD3. ${ }^{5,6}$ For patients with ACTA2 mutations, aortic events are more prevalent in men than in women ( $62 \%$ vs $40 \%)$; however, age at first aortic event was similar between the sexes. ${ }^{7}$ These data suggest that sex differences exist and should be further investigated among patients with genetically triggered TAAD.

For patients with TAAD without a clear genetic etiology, the prevalence of thoracic aortopathy is also higher in men, who account for nearly $70 \%$ of individuals with TAAD. ${ }^{8}$ However, this male predisposition is significantly attenuated in older individuals, who predominantly have degenerative thoracic aortic disease. ${ }^{8}$ Despite the lower predisposition to develop aortopathy, women with thoracic aortic aneurysm (TAA) are 3 times more likely to experience dissection or rupture, ${ }^{9,10}$ especially at smaller aneurysm sizes, ${ }^{11}$ and are $40 \%$ more likely than men to die from TAA. ${ }^{11,12}$ Thus, although women appear to be "naturally protected" against thoracic aortic disease, in those women who go on to develop the condition, this may be a signal that their aortas harbor a greater burden of wall pathology and/or are exposed to greater hemodynamic challenges-all of which may contribute to worse aorta-related outcomes in women.

Although women are often older and have more comorbidities than men by the time they develop TAA, studies have shown that the poor outcomes in women are independent of age and comorbidities. Several authors have supported the notion of relative aortic size disadvantaging women, who are usually smaller than men with respect to height and baseline aortic diameter. Forbes and colleagues $^{13}$ confirmed this by demonstrating that a TAA diameter of $4.97 \mathrm{~cm}$ in a woman correlates to a $6.0-\mathrm{cm}$ TAA in a man. Similarly, Davies and colleagues ${ }^{14}$ found that indexing aneurysm to body size was more accurate than absolute diameter for predicting dissection. Thus, it is important for clinicians to consider aortic size with respect to body size when making clinical decisions for the management of TAA, which may result in earlier intervention and improvement of TAA outcomes in women. However, in the same study by Davies and colleagues, ${ }^{14}$ female sex still emerged as an independent risk factor for acute aortic syndromes despite indexing aneurysm size to body size. These results corroborate the notion that sex differences in TAA outcomes are complex and cannot be attributed simply to differences in body size between men and women.

The aging nonaneurysmal aorta remodels differently in men and women. Although men's aortas are larger to begin with, the dynamics of aortic growth in later life are greater in women. Accordingly, we have demonstrated that aneurysm expansion is 3 times faster in women than in men with TAA once aneurysm is indexed to body size, ${ }^{12}$ a finding that was subsequently confirmed by the Yale group. ${ }^{15}$ These sex differences in TAA growth were only observed in people with degenerative TAAs (typically older and with high prevalence of hypertension), however, suggesting an effect of arterial aging on the adverse TAA behavior in women. Although sex differences in aortic behavior shed further light on sex differences in TAA outcomes, their underlying mechanisms remain poorly understood.

One possibility lies in the molecular and hemodynamic differences between men and women. We have demonstrated that aortic stiffness (a robust marker of aortic health and arterial aging) is a better correlate of TAA expansion in women than in men. ${ }^{16}$ Corroborating this notion on a molecular level, Sokolis and colleagues ${ }^{17}$ reported higher levels of matrix metallopeptidase (MMP)-2 and MMP-9 (enzymes capable of degrading and remodeling the arterial wall) and decreased expression of the inhibitory enzymes tissue inhibitors of metalloproteinase (TIMP)-1 and TIMP-2 in women than men. This impairment of aortic wall homeostasis, resulting in enhanced extracellular matrix degradation, leads to a higher aortic elastic modulus, increased aortic stiffness, and decreased aortic strength in women. These reported abnormalities in aortic wall health and biomechanics are likely to have direct links to the worse aortic hemodynamics in women. Accordingly, it is now well established that aortic hemodynamics deteriorate at a faster rate in aging women compared with men, ${ }^{18}$ leading to worse pulsatile hemodynamics in women after age 50 . This in turn directly contributes to the greater prevalence of hypertension, especially isolated systolic hypertension, in older women. Because hypertension is associated with a 2.6-fold greater odds of aortic dissection, is highly prevalent in patients with $\mathrm{TAAD},{ }^{19}$ and has the highest population-attributable risk for dissection $(54 \%),{ }^{19}$ older women's greater predisposition to adverse arterial hemodynamics and hypertension likely contributes significantly to their faster rates of aneurysm expansion and sex differences in outcomes.

In summary, aging appears to affect aortic health and structure more adversely in women than in men, characterized by greater extracellular matrix remodeling, more rapid alterations in aortic geometry, and worse pulsatile hemodynamics in women. Thus, the development of TAA in older women may cause other abnormalities in aortas that are already structurally and functionally compromised, leading to further deterioration in aortic 
health and potentially explaining women's faster aneurysm expansion and worse TAAD-related outcomes.

\section{Sex Differences in Surgical Outcomes Following TAA Repair}

Our recent study, "Sex-Related Differences in Patients Undergoing Thoracic Aortic Surgery," published in Circulation, evaluated the impact of sex on outcomes following aortic arch surgery. ${ }^{20}$ The study cohort comprised 1653 patients (30\% female) undergoing arch repair at 10 institutions in the Canadian Thoracic Aortic Collaborative. Female patients were older and presented with larger indexed aortic diameters, whereas male patients had more coronary disease and lower ejection fractions. The duration of and cerebral protection strategies used during circulatory arrest were similar in the sexes; however, male patients underwent more complex root operations, resulting in longer cardiopulmonary bypass times. Our major finding was that despite undergoing less complex operations, females experienced higher rates of mortality $(11 \%$ vs $7.4 \% ; P=.02)$, stroke $(8.8 \%$ vs $5.5 \% ; P=.01)$, and a composite of in-hospital adverse events ( $31 \%$ vs $27 \% ; P=.04)$. After accounting for differences in baseline characteristics, female sex was identified as an independent predictor of death (odds ratio [OR], 1.81; 95\% confidence interval [CI], 1.24-1.89; $P<.001)$, stroke (OR, 1.90; 95\% CI, 1.28-2.85; $P<.001$ ), and adverse events (OR, 1.40; 95\% CI, 1.16-1.69; $P<.001)$. These findings were consistent among patients undergoing elective surgery and those undergoing emergency surgery.

The vast majority of emergency arch operations are for acute aortic dissection, and sex-based differences have also been observed by the International Registry of Acute Aortic Dissection (IRAD). Data from IRAD on 549 patients who underwent surgery for acute type A dissection demonstrated higher mortality in women following surgical repair $(31.9 \%$ in women vs $21.9 \%$ in men; $P=.013){ }^{8}$ Women presented at an older age, were diagnosed later, and presented with higher rates of coma, hypotension, and tamponade. The late presentation and increased complications at diagnosis likely contributed to the poor outcomes observed. A more recent study further substantiated this finding and also reported a significant delay in the diagnosis of acute aortic dissection in women. ${ }^{21}$ Similar to observations in patients with acute coronary syndrome, women were less likely than men to experience the typical acute onset of chest pain, likely contributing to the later diagnosis.

In addition to worse outcomes following surgical repair, the IRAD study found that fewer women underwent surgery, which is the gold standard treatment for acute type A dissection. These findings echo research in the field of abdominal aortic aneurysms (AAA), where women have also been found to have lower repair rates and thus worse outcomes. ${ }^{22}$ Female sex has been associated with a faster growth rate of AAA and rupture at smaller diameters compared with men, with one study reporting a mean $1-\mathrm{cm}$ difference in aortic diameter at the time of rupture. ${ }^{23}$ What is unproven is whether there exists bias from surgeons regarding perceived poor outcomes for women that may partially explain the lower repair rates for acute type A dissection or ruptured AAA. Interestingly, there were no sex-related differences in the management or outcomes of acute type B aortic dissections, which are typically medically treated. ${ }^{8}$

The sex-related differences in outcome following elective arch repair in our study may be related to a number of factors. The increased age and greater indexed aortic diameter at the time of repair for women implies more advanced disease at the time of surgery. This pattern of later referral and presentation has been observed repeatedly in coronary, valve, and heart failure surgery. Furthermore, despite having larger indexed aortic diameters, women in our study underwent less complex and potentially less comprehensive aortic repairs compared with men. Suboptimal management of disease pathology once it has been identified also has been shown for coronary, valvular, and abdominal aortic disease. The poor surgical outcomes that we observed also may be related to possibly more fragile vessels that require technically more demanding operations in women. Other proposed mechanisms include the lower total body blood volume in women, leading to higher transfusion rates and worse outcomes. The explanation is likely multifactorial, culminating in women having a lower physiological reserve for surgical insults.

Given these observations, it is possible that women may derive disproportionately more benefit from less invasive procedures that mitigate surgical insult. To support this hypothesis, a recent meta-analysis of transcatheter versus surgical aortic valve replacement trials found that women experienced lower mortality when undergoing a transcatheter approach compared with conventional surgery, in contrast to men, who had equivalent outcomes. ${ }^{24}$ In the endovascular aneurysm repair (EVAR) literature, although women have higher mortality than men following open AAA repair, this gap is diminished for patients undergoing EVAR, although complication rates remain higher for women. ${ }^{25}$ Improvements in surgical technique for aortic arch repair that minimize hypothermia and circulatory arrest, such as the routine use of antegrade cerebral perfusion, may disproportionately benefit female patients and close the observed outcome gap in our study. Hybrid arch grafts and other advanced endovascular approaches have been developed to treat arch pathology. These promising less invasive approaches may be of particular benefit to women at high risk for open arch repair. 
Interestingly, the pattern of women benefiting from less invasive surgical techniques is not seen in studies evaluating differences between the sexes for the descending thoracic and thoracoabdominal aorta. Two large retrospective single-center series of open repair conducted at Baylor and Cornell reported highly congruent findings. ${ }^{26,27}$ Women in both studies were older with more pulmonary disease and more frequently presented with degenerative aneurysms rather than dissections. To address these baseline differences, the Baylor study used propensity score matching and the Cornell study used multivariable logistic regression. Both studies found that female patients had the same operative mortality as male patients but had a higher rate of respiratory complications. The Cornell group found that female sex predicted worse long-term survival. ${ }^{27}$ Meanwhile, Deery and colleagues ${ }^{28}$ evaluated data from the Society for Vascular Surgery Vascular Quality Initiative (SVS-VQI) registry to study sex-related differences in endovascular repair of descending TAAs. Here again, the women were older and had larger indexed aortic sizes, more pulmonary disease, and less coronary disease. However, the women experienced longer hospitalizations, as well as higher 30-day and 1-year mortality rates. After multivariable logistic regression, female sex was found to independently predict both perioperative and long-term mortality. Similarly, a multicenter German study on endovascular repair of thoracoabdominal aneurysms using branched and fenestrated grafts also identified female sex as an independent predictor of perioperative and long-term mortality. ${ }^{29}$

More replicative studies are needed to better understand the phenomena of sex-related surgical outcome differences. We advocate for close attention to sex-specific factors and reporting as we design future studies and gain experience with emerging less invasive technologies.

\section{Conclusions and Future Directions}

As we have summarized, there is growing evidence supporting altered and less favorable biology of the aortic wall in women, particularly as they age. Women have been observed to have higher rates of aortic growth, rupture, and dissection at smaller aortic diameters, as well as delayed presentation and diagnosis of acute dissection. Furthermore, women fare worse with surgical repair after both elective and emergency surgery for thoracic aortic pathology.

To this end, we propose a call to action to (1) ensure incorporation of sex and gender in all TAAD research, including natural history studies; (2) further explore nonsize-related mechanisms for sex differences in TAA, including differences in aortic aging, structure, and function; and (3) develop and validate novel sex-based algorithms to crystallize risk stratification, disease monitoring, treatment strategies, and prevention of complications in TAAD. Figure 1 summarizes the sex differences in the biology, presentation, management, and outcomes of patients with thoracic aortic disease.

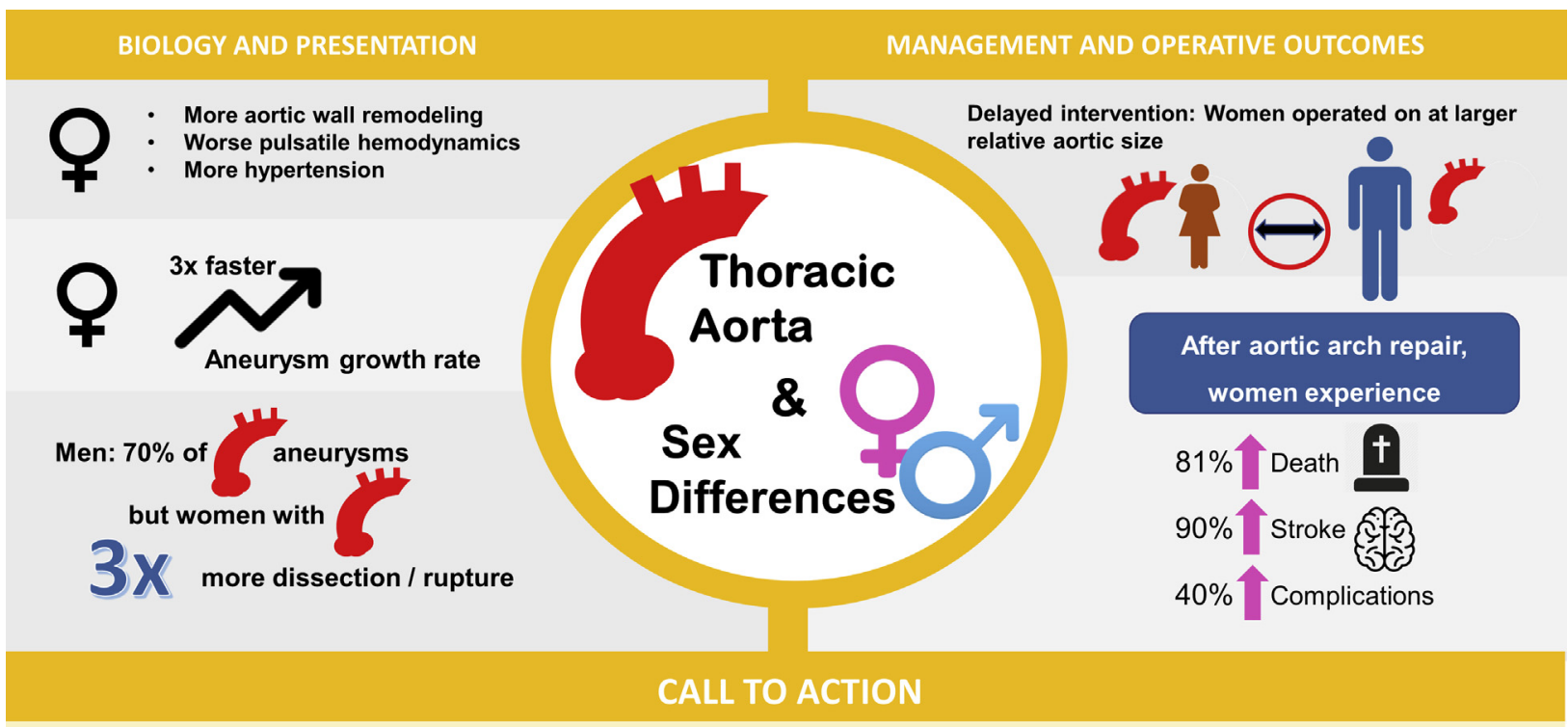

1. INCORPORATE SEX AND GENDER INTO ALL RESEARCH ON THORACIC AORTIC DISEASE

2. INVESTIGATE MECHANISMS OF SEX DIFFERENCES IN THORACIC AORTIC AGING, STRUCTURE AND FUNCTION

3. DEVELOP SEX-BASED ALGORITHMS FOR RISK STRATIFICATION, MONITORING AND TREATMENT

FIGURE 1. The thoracic aorta and sex differences. Sex differences exist in the biology, presentation, management, and outcomes of patients with thoracic aortic disease. 
In the abdominal aortic literature, the accumulating evidence of worse outcomes in women has led to sex-specific recommendations of diameter threshold for elective repair, with repair offered at $5.5 \mathrm{~cm}$ in men and $5.0 \mathrm{~cm}$ in women. There is a pressing need to refine the current thoracic aortic guidelines to incorporate sex-specific variations in aortic size and body surface area to inform thresholds for elective surgery in TAAD. Clinicians should consider the distinct constellation of symptoms that women with acute dissection may harbor. Natural history data on TAAD remain sparse, and further research is needed to develop and validate markers other than size to identify vulnerable aortas and predict rupture. Similarly, further study is needed in women with TAAD contemplating pregnancy, to improve the management of this high-risk population.

We encourage sex-based analysis of large national and international registries of thoracic aneurysms and dissections. Similarly, prospective studies of TAAD should be deliberate in their design and conduct to identify differences in outcomes for men and women. The complex interactions among genetic, hormonal, and environmental aspects of TAAD development require further study to elucidate the mechanisms behind these observed differences. As sexspecific data on the presentation, etiology, and outcomes of TAAD accumulate, we will be better equipped to provide personalized management of patients with thoracic aortic disease.

\section{Conflict of Interest Statement}

Authors have nothing to disclose with regard to commercial support.

\section{References}

1. Olsson C, Thelin S, Ståhle E, Ekbom A, Granath F. Thoracic aortic aneurysm and dissection: increasing prevalence and improved outcomes reported in a nationwide population-based study of more than 14,000 cases from 1987 to 2002. Circulation. 2006;114:2611-8.

2. Détaint D, Faivre L, Collod-Beroud G, Child AH, Loeys BL, Binquet C, et al Cardiovascular manifestations in men and women carrying a FBN1 mutation. Eur Heart J. 2010;31:2223-9.

3. Roman MJ, Devereux RB, Preiss LR, Asch FM, Eagle KA, Holmes KW, et al. Associations of age and sex with Marfan phenotype: the National heart, lung, and blood institute GenTAC (Genetically triggered thoracic aortic aneurysms and cardiovascular conditions) registry. Circ Cardiovasc Genet. 2017;10:e001647.

4. Meijboom LJ, Timmermans J, Zwinderman AH, Engelfriet PM, Mulder BJ. Aortic root growth in men and women with the Marfan's syndrome. Am J Cardiol. 2005;96:1441-4

5. Jondeau G, Ropers J, Regalado E, Braverman A, Evangelista A, Teixedo G, et al. International registry of patients carrying TGFBR1 or TGFBR2 mutations: results of the MAC (Montalcino Aortic Consortium). Circ Cardiovasc Genet. 2016;9:548-58

6. Hostetler EM, Regalado ES, Guo DC, Hanna N, Arnaud P, Muiño-Mosquera L, et al. SMAD3 pathogenic variants: risk for thoracic aortic disease and associated complications from the Montalcino Aortic Consortium. J Med Genet. 2019;56: 252-60.

7. Regalado ES, Guo DC, Prakash S, Bensend TA, Flynn K, Estrera A, et al. Aortic disease presentation and outcome associated with ACTA2 mutations. Circ Cardiovasc Genet. 2015;8:457-64.
8. Nienaber CA, Fattori R, Mehta RH, Richartz BM, Evangelista A, Petzsch M, et al. Gender-related differences in acute aortic dissection. Circulation. 2004; 109:3014-21.

9. Juvonen T, Ergin MA, Galla JD, Lansman SL, Nguyen KH, McCullough JN, et al. Prospective study of the natural history of thoracic aortic aneurysms. Ann Thorac Surg. 1997;63:1533-45.

10. Davies RR, Goldstein LJ, Coady MA, Tittle SL, Rizzo JA, Kopf GS, et al. Yearly rupture or dissection rates for thoracic aortic aneurysms: simple prediction based on size. Ann Thorac Surg. 2002;73:17-27 [discussion: 27-8].

11. Pape LA, Tsai TT, Isselbacher EM, Oh JK, O'Gara PT, Evangelista A, et al Aortic diameter $>$ or $=5.5 \mathrm{~cm}$ is not a good predictor of type A aortic dissection: observations from the International Registry of Acute Aortic Dissection (IRAD) Circulation. 2007;116:1120-7.

12. Cheung K, Boodhwani M, Chan KL, Beauchesne L, Dick A, Coutinho T. Thoracic aortic aneurysm growth: role of sex and aneurysm etiology. J Am Heart Assoc. 2017;6:e003792

13. Forbes TL, Harris JR, Lawlor DK, Derose G. Evaluation of sex differences in relative dilatation of thoracic aortic aneurysms. Eur J Vasc Endovasc Surg. 2010;39:555-8

14. Davies RR, Gallo A, Coady MA, Tellides G, Botta DM, Burke B, et al. Novel measurement of relative aortic size predicts rupture of thoracic aortic aneurysms. Ann Thorac Surg. 2006;81:169-77.

15. Zafar MA, Li Y, Rizzo JA, Charilaou P, Saeyeldin A, Velasquez CA, et al. Height alone, rather than body surface area, suffices for risk estimation in ascending aortic aneurysm. J Thorac Cardiovasc Surg. 2018;155:1938-50.

16. Boczar KE, Cheung K, Boodhwani M, Beauchesne L, Dennie C, Nagpal S, et al. Sex differences in thoracic aortic aneurysm growth. Hypertension. 2019;73:190-6.

17. Sokolis DP, Iliopoulos DC. Impaired mechanics and matrix metalloproteinases/ inhibitors expression in female ascending thoracic aortic aneurysms. J Mech Be hav Biomed Mater. 2014;34:154-64.

18. Mitchell GF, Wang N, Palmisano JN, Larson MG, Hamburg NM, Vita JA, et al. He modynamic correlates of blood pressure across the adult age spectrum: noninvasive evaluation in the Framingham Heart Study. Circulation. 2010;122:1379-86.

19. Landenhed M, Engström G, Gottsäter A, Caulfield MP, Hedblad B, Newton-Cheh C, et al. Risk profiles for aortic dissection and ruptured or surgically treated aneurysms: a prospective cohort study. J Am Heart Assoc 2015;4:e001513.

20. Chung J, Stevens LM, Ouzounian M, El-Hamamsy I, Bouhout I, Dagenais F, et al. Sex-related differences in patients undergoing thoracic aortic surgery. Circulation. 2019;139:1177-84.

21. Harris KM, Strauss CE, Eagle KA, Hirsch AT, Isselbacher EM, Tsai TT, et al. Correlates of delayed recognition and treatment of acute type A aortic dissection: the International Registry of Acute Aortic Dissection (IRAD). Circulation. 2011; 124:1911-8.

22. Dueck AD, Johnston KW, Alter D, Laupacis A, Kucey DS. Predictors of repair and effect of gender on treatment of ruptured abdominal aortic aneurysm. $J$ Vasc Surg. 2004:39:784-7.

23. Evans SM, Adam DJ, Bradbury AW. The influence of gender on outcome after ruptured abdominal aortic aneurysm. J Vasc Surg. 2000;32:258-62.

24. Panoulas VF, Francis DP, Ruparelia N, Malik IS, Chukwuemeka A, Sen S, et al. Female-specific survival advantage from transcatheter aortic valve implantation over surgical aortic valve replacement: meta-analysis of the gender subgroups of randomised controlled trials including 3758 patients. Int J Cardiol. 2018;250:66-72.

25. Chung C, Tadros R, Torres M, Malik R, Ellozy S, Faries P, et al. Evolution of gender-related differences in outcomes from two decades of endovascular aneurysm repair. J Vasc Surg. 2015;61:843-52.

26. Spiliotopoulos K, Price MD, Amarasekara HS, Green SY, Zhang Q, Preventza O, et al. Are outcomes of thoracoabdominal aortic aneurysm repair different in men versus women? A propensity-matched comparison. J Thorac Cardiovasc Surg. 2017;154:1203-14.e6.

27. Girardi LN, Leonard JR, Lau C, Ohmes LB, Gambardella I, Iannacone EM, et al. Gender-related outcomes after open repair of descending thoracic and thoracoabdominal aortic aneurysms. J Vasc Surg. 2019;69:1028-35.e1.

28. Deery SE, Shean KE, Wang GJ, Black JH III, Upchurch GR Jr, Giles KA, et al. Female sex independently predicts mortality after thoracic endovascular aortic repair for intact descending thoracic aortic aneurysms. J Vasc Surg. 2017;66:2-8.

29. Rieß HC, Debus ES, Schwaneberg T, Sedrakyan A, Kölbel T, Tsilimparis N, et al Gender disparities in fenestrated and branched endovascular aortic repair. Eur J Cardiothorac Surg. 2019;55:338-44. 